\title{
Evolutionary Daisyworld models: A new approach to studying complex adaptive systems
}

\author{
Juan Carlos Nuño ， Javier de Vicente , José Olarrea , Pilar López , Rafael Lahoz-Beltrá \\ Department of Mathenatics Applied to Natural Resources, Escuela Técnica Superior de Ingenieros de Montes, Universidad Rolitécnića de Madrid, 28040-Madrid, Spain \\ Department of Applied Mathematics and Statistics, Escuela Técnica Superior de ingenieros Aeronáticos, Universidad Politécnica de Madrid, 28040-Madrid, Spoin \\ Department of Applied Mathematics, Faculty of Biological Sciences, Universidad Complutense de Madrid. 28040-Madrid. Spoin
}

\author{
Keywords: \\ Mathematical modelling \\ Complex adaptive system \\ Evolutionary algorithm \\ Daisywolld \\ Quasispecies
}

\begin{abstract}
A B S T R A C T
This paper presents a model of a population of error-prone self-replicative species (replicators) that interact with its environment. The population evolves by natural selection in an environment whose change is caused by the evolutionary process itself. For simplicity, the environment is described by a single scalar factor, i.e. its temperature. The formal formulation of the model extends two basic models of Ecology and Evolutionary Biology, namely, Daisyworld and Quasispecies models. It is also assumed that the environment can also change due to external perturbations that are summed up as an external noise. Unlike previous models, the population size self-regulates, so no ad hoc population constraints are involved. When species replication is error-free, i.e. without mutation, the system dynamics can be described by an $(n+1)$-dimensional system of differential equations, one for each of the species initially present in the system, and another for the evolution of the environment temperature. Analytical results can be obtained staightforwardly in lowdimensional cases. In these examples, we show the stabilizing effect of themal white noise on the system behavior. The error-prone self-replication, i.e. with mutation, is studied computationally. We assume that species can mutate two independent parameters: its optimal growth temperature and its inlluence on the environment temperature. For different mutation rates the system exhibits a large variety of behaviors. In particular, we show that a quasispecies distribution with an internal sub-distribution appears, facilitating species adaptation to new environments. Finally, this ecologically inspired evolutionary model is applied to study the origin and evolution of public opinion.
\end{abstract}

\section{Introduction}

An ecosystem is, by definition, a system formed by interacting species where a strong coupling between these and the environment also exists. The influence of the environment perturbation on system evolution has often been neglected in fields such as biology or sociology. Both Ecology and Biology need to consider evolution as a fundamencal issue. However, evolution has been mainly handled by evolutionary biologists whereas ecologists mainly focused on already formed (eco)-systems. Fortunately, eco-informatics approaches have started to connect both fields each other (Hogeweg, 2007; Whigham and Fogel, 2006).

The problem of evolution and selection of a population of noninteracting error-prone self-replicative species (replicators) was already addressed in the seventies by Eigen and coworkers (Eigen, 1971; Eigen et al., 1989; Schuster and Sigmund, 1983). The classical formulation of this model does not consider any interaction between species and the environment, and so the self-replicative fitness of each species is constant in time. On the other hand, this interaction is included in the Daisyworld model of Watson and Lovelock as a key ingredient to explain the self-regulatory capabilities of our planet (Lovelock, 1992; Watson and Lovelock, 1983). Nonetheless, this seminal ecological model does not take into account species evolution by natural selection. In the classical Daisyworld daisies self-regulate the global temperature that is continuously raised by solar radiation. Both models were originally formulated as dynamical systems (in terms of Ordinary Differential Equations) and are combined in this paper to study biological evolution in an ecological framework (Table 1).

The interaction between the species and the environment has an imporcant effect on the species reproductive fitness: it changes as the environment is modified by the current population. The fitness landscape is then intrinsically dynamic. Although originally stated by Eigen (1971), few papers have addressed dynamic landscapes caused by species interactions (Bak and Sneppen, 1993) and, more recently, external perturbations (Nilsson and Snoad, 2000; Rauch et al., 2002; Saakian and $\mathrm{Hu}, 2006$ ). The problem here is quite different, since the fitness landscape varies as a consequence of mutation. Mutation is the driving force for evolution, not only of the population but also of its 
Table 1

In this table we compare four different dynamical systems that differ in the number of factors they take into account. The replicator equation resulting in Molecular Evolution theory describes a constant population of error-free self-replicative species. The replicator equation is modified to include mutation, i.e. error-prone self-replication. Thus, the population is able to evolve and search for bettri replicators (see, for instance, Eigen, 1971: Eigen and Schuster, 1979: Eigen et al., 1989: Schuster and Sigmund, 1983). In both inodels the replicator growth rate is independent of the environment. In other words, the enviromment is assumed to be fixed and constant in time. The classical Daisyworld of Watson and Lovelock assumes a population of non-interacting species (daisies) in a changing environment (due to the increasing radiation flux from the Sun). In this model, daisies modify their local temperature and thus self-regulate the global temperature (Lovelock, 1992; Watson and Lovelock, 1983). If error-prone selfreplication is assumed, then an evolutionary process takes place in a Daisyworld within a changing environment (Lansing et al., 1998; McDonald-Gibson et al., 2008). This is the colnplete dynamical system we consider in this paper.

\begin{tabular}{lllll}
\hline & Mutation & $\begin{array}{l}\text { Species } \\
\text { intraction }\end{array}$ & $\begin{array}{l}\text { Species-environment } \\
\text { interaction }\end{array}$ & $\begin{array}{l}\text { Population } \\
\text { size constraint }\end{array}$ \\
\hline $\begin{array}{c}\text { Replicator dynamics } \\
\text { Replicator-mutation }\end{array}$ & NES & NO & NO & YES \\
$\begin{array}{c}\text { dynamic } \\
\text { Daisyworld }\end{array}$ & NO & NO & NO & YES \\
$\begin{array}{c}\text { Evolutionary } \\
\text { Daisyworld }\end{array}$ & YES & NO & YES & YES \\
\hline
\end{tabular}

environment. Besides the change in the environment by the action of the species, there may be an extrinsic variation due to other external factors. Despite the possible existence of a trend (e.g. seasonal variations, climate warming or an increase in $\mathrm{CO}_{2}$ ) our model includes a number of uncorrelated perturbations whose global effect, according to the Central Limit Theorem is a Gaussian white noise (Gardiner. 1995).

As we stated before, this global approach is not exclusive to evolutionary ecology. The interaction between agents and the environment is also fundamental in sociology. The model defined in this paper is applied to study the formation and evolution of social opinions (Alves et al., 2002; Bagnoli et al., 2002; Tipan Verella and Wardak, 2008; Van Ginneken, 2003). In this sense, this ecologically inspired evolutionary algorithm can be viewed as a problem solving tool available to other disciplines.

This paper is organized as follows. The next section presents the quasispecies world based on both the Daisyworld and the quasispecies models. Error-free (no mutation) systems that can be described by a $n+1$-dimensional stochastic dynamical system are presented in subsection 3.1. In particular, we handle low-dimensional cases and the effect of external noise. In Section 4 , an evolutionary algorithm is built to study the formation and evolution of public opinion. The paper ends with a discussion about the fundamentals and possible applications of this model.

\section{The quasispecies-world model}

Let us consider a system formed by non-interacting error-prone self-replicative species (replicators) I, whose population at time $t$ is given by $N_{i}(t)$. The total population at time $t$

$N(t)=\sum_{i=1} N_{i}(t)$

is generally not constant in time and, as will be seen later, always remains bounded.

In the process of self-replication each species $I_{\text {; }}$ can mutate into a new species $I_{j}$ with probability $Q_{i j}$. The so called mutation matrix $Q=$ $\left[Q_{i j}\right]$ drives the evolutionary properties of the system. Furthermore, each species $l_{i}$ is assumed to have a phenotypic representation that can be described by two real numbers: $T_{i}$ and $\alpha_{i}$. The first one defines its optimal growth "temperature", whereas the second one measures its influence on the environment. Both parameters $T_{i}$ and $\alpha_{i}$ define the survival probability of species $I_{i}$ in each generation, the so called amplification factor or fitness function, $f_{i}$. Contrary to the classical Eigen model, at each moment $f_{i}$ depends on the rest of species of the population trough the temperature global temperature $T$. In particular, we assume that the reproductive fitness of copy $l_{i}$ is given by a Gaussian curve centered in $T_{i}$. Formally,

$f_{i}(T)=r_{i} e^{-a_{4}\left(T-T_{1} r^{2}\right.}$

where $r_{i}$ and $a_{i}$ are non-negative parameters. Note that the first order Taylor expansion is an inverted parabola as used in previous Daisyworld models (Lovelock, 1992; Saunders, 1994; Watson and Lovelock, 1983; Wood et al., 2008).

The intensive parameter $T$, a sort of global temperacure, characterizes the environment. It changes over time as the population evolves. It is assumed that each copy $I_{i}$ has a linear influence on $T$, weighted by the real parameters $\alpha_{i}$. In addition, external perturbations can modify the value of $T$. Thus, the time evolution of the global temperature is given by:

$\dot{T}=\sum_{i=1}^{N} \alpha_{i} x_{i}-T+\xi_{r}$

here $\dot{T}$ means the derivative of $T$ with respect to $t$. As stated in the introduction, the noise $\xi_{t}$ is assumed to be Gaussian white noise with zero mean, i.e. an uncorrelated process satisfying:

$\left\langle\xi_{c}\right\rangle=0 \quad\left\langle\xi_{t} \xi_{s}\right\rangle=\delta(t-s)$

where $\langle$ means expected value (Gardiner, 1995). Note that, in the absence of any replicative species, the reference value of $T$ fluctuates around $T_{0}=0$ (since no trend in the external noise exists).

The time evolution of the population is described by a system of Ordinary Differential Equations of variable dimension (the number of species changes due to mucation). In particular, the species population size $x_{i}$ changes over time according to:

$\dot{x}_{i}=\sum_{j} x_{j} Q_{j i} f_{j}(T)-x_{i} \delta_{i}$

where $\delta_{i}$ is the death rate of $I_{i}$ that, for the sake of simplicity, is considered to be independent of $T$. If the species replication is errorfree, i.e. $Q_{i i}=1$ and $Q_{i j}=0$, for all $i \neq j$ then, this equation reduces to the much simple form:

$\dot{x}_{i}=x_{i}\left(f_{i}(T)-\delta_{i}\right)$

for $i=1,2, \ldots, N$.

In the classical quasispecies model, where the amplification factor is independent of $T$, selection can only occur if additional constraints are imposed (e.g. constant population). In this case, the species with the largest productivity $E_{i}=f_{i}-\delta_{i}$ is selected and occupies the whole population. On the contrary, if there are no limits to the population size, those species with positive productivity, i.e. $E_{i}>0$ would tend to infinity, whereas species with negative productivity will become extinct. If the productivity depends on $T$ and species influence on $T$ is not null (i.e. $\alpha \neq 0$ ), then the total population is bounded.

\section{Error-free low-dimensional models}

The system of stochastic differential Eqs. (6) and (3) governs the time evolution of a population of replicators of variable size in a changing environment. Through $T$, the species amplification factor $f_{i}$ changes over time and so does the whole fitness landscape. Unfortunately, the complexity of this system makes any analytical study difficult. However, some interesting insights can be obcained from the analysis of particular situations. 


\subsection{How does a unique replicator modify its environment?}

The simplest case occurs when only one species exists. Let us also assume that no external noise aflects the global temperature. The time evolution of this system is given by:

$\dot{x}=x\left(r e^{-\alpha ! T-T m !^{2}}-\delta\right)$

$\dot{T}=\alpha x-T$

where $a, \delta$ and $r$ are positive real parameters. Parameter $\alpha$ can take null, negative or positive values, meaning that the global temperature $T$ can be either unaffected, decreased or increased, respectively by the presence of the unique species.

The equilibrium states of this dynamical system can be obtained equating to 0 the right hand sides of $\mathrm{Eq}$. (7). One equilibrium solution that exists for all parameter values is $\bar{x}=0$ and, consequently $\bar{T}=0$. This means species extinction. Since $r e^{-a\left(T-T_{m}\right)^{2}} \leq 1$, no additional equilibrium solutions exist if $\delta>r$. Therefore, in this case, the extinction state is a global attractor. On the contrary, when $\delta<r$, two additional equilibrium solutions of Eq. (7) exist for $\alpha \neq 0$. The equilibrium populations are given by:

$\bar{x}_{p, m}=\frac{T_{m} \pm T_{c}}{\alpha}$.

These two solutions are positive only if $\left|T_{m}\right|>T_{c}$, where the critical temperature is given by:

$T_{c}=\sqrt{-\frac{1}{a} \ln \left(\frac{\delta}{r}\right)}$

When a positive equilibrium population exists, then the system reaches the equilibrium temperature:

$\bar{T}_{p, m}=\alpha \bar{x}_{p, m}$.

This stationary temperature is either positive or negative depending on the value of $\alpha$

By linearization around the steady scate (Hirsch and Smale, 1974), it is straightforward to prove that if $\left|T_{n n}\right|>T_{c}$ the extinction state is asymptotically stable and the upper branch of Eq. (8) is asymptotically scable (the two eigenvalues of the Jacobian matrix are negative), whereas the lower one is unscable (one of the eigenvalues is positive). In this case, a bistable situation occurs between extinction and the higher branch $\bar{x}_{p}$ (see Fig. 1 ).

\subsection{Competition and adaptation in the 2-species replicator model}

As has been shown in the previous section, a given species is able to shift the global temperature to the new value (10) that depends on the species characteristics, namely $\alpha$ and $T_{m}$. In this section we study the behavior of two species that live in the same (eco)system. As before, each species $I_{i}$ is described by its optimal growth temperature $T_{i}$ and its influence on the global temperature $\alpha_{i}$. Let $x_{1}$ and $x_{2}$ be the population size of species $I_{1}$ and $I_{2}$, respectively and let $T$ be the global temperature of the system. As before, no external noise is considered. A fitness function, as given by Eq. (2), is associated with each species. The death rate $\delta_{i}$ for each species, $i=1,2$ is supposed to be independent on T. In this case, the dynamical system (6) and (3) reduces to:

$\dot{x}_{1}=x_{1}\left(f_{1}(T)-\delta_{1}\right)$

$\dot{x}_{2}=x_{2}\left(f_{2}(T)-\delta_{2}\right)$

$\dot{T}=\alpha_{1} x_{1}+\alpha_{2} x_{2}-T$.

We start the qualitative analysis of this system by computing its steady states. As in the previous one species model, extinction

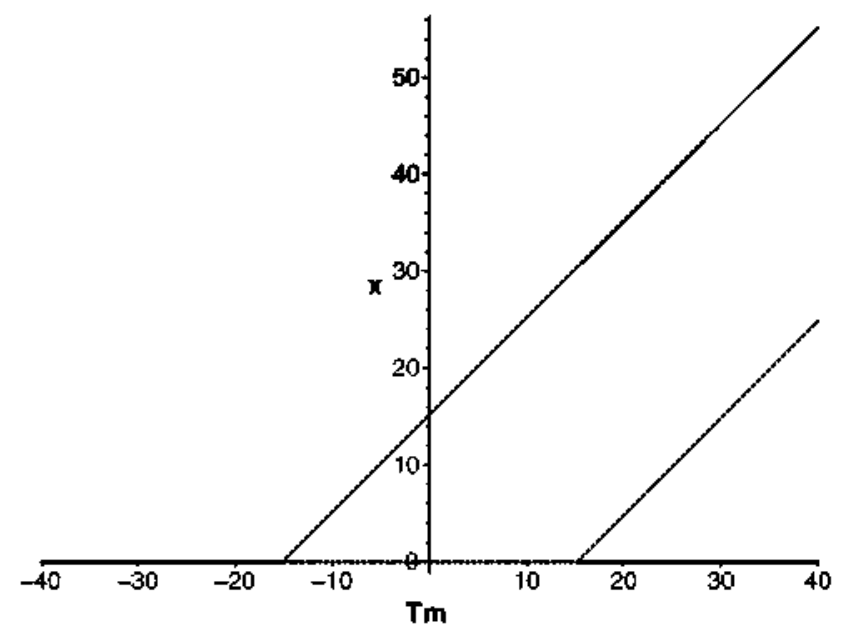

Fig. 1. Bifurcation diagram for system (7). Parameter values are: $a=0.01 ; \delta=0.1$; $\alpha=0.01 ; r=1$. The critical temperature is $T_{\mathrm{c}} \approx 15.1743$. Notice that the total population size at equilibium is bounded. In the case of having $\alpha<0$, the resulting bifurcation diagram would be a reflection with respect to the vertical axis. In solid line, the stable higher branch was shown. In dashed line, the unstable lower branch was shown.

$\left(\bar{x}_{1}=\bar{x}_{2}=0\right.$ and $\left.\bar{T}=0\right)$ always exists, for all values of the parameters. Two additional steady scates, which means the selection of one of the species, can also appear.

Let us assume for instance, that $\bar{x}_{1}=0$. The stationary values of both $\bar{x}_{2}$ and $\bar{T}$ are, then, obtained by solving the algebraic system:

$f_{2}(\bar{T})=\delta_{2}$

$\bar{T}=\alpha_{2} \bar{x}_{2}$

that gives rise, for $\alpha_{2} \neq 0$, to a similar steady value as in Eq. (8):

$\bar{x}_{2}=\frac{T_{2} \pm T_{c 2}}{\alpha_{2}}$

where

$T_{c 2}=\sqrt{-\frac{1}{a_{2}} \ln \left(\frac{\delta_{2}}{r_{2}}\right)}$.

Obviously, there is a symmetric steady state with $\bar{x}_{2}=0$ that is obtained from the algebraic system:

$f_{1}(\bar{T})=\delta_{1}$

$\bar{T}=\alpha_{1} \bar{x}_{1}$.

If, $\alpha_{1} \neq 0$, it is given by:

$\bar{x}_{1}=\frac{T_{1} \pm T_{c 1}}{\alpha_{1}}$

with

$T_{c 1}=\sqrt{-\frac{1}{a_{1}} \ln \left(\frac{\delta_{1}}{r_{1}}\right)}$.

The coexistence of both species requires that

$T_{1} \pm \sqrt{-\frac{\ln \left(\frac{o_{1}}{r_{1}}\right)}{a_{1}}}=T_{2} \pm \sqrt{-\frac{\ln \left(\frac{o_{2}}{r_{2}}\right)}{a_{2}}}$.

This is a degenerate case that gives rise to the family of straight lines formed by scationary points $\alpha_{1} \bar{x}_{1}+\alpha_{2} \bar{x}_{2}=\tau$, for any real parameter $\tau$. 
As before, the scability analysis can be performed by linearization (see, for inscance, Hirsch and Smale, 1974). In this case, the Jacobian is the 3-dimensional matrix:

$J=\left[\begin{array}{ccc}r_{1} e^{-a_{1}\left(T-T_{1}\right)^{2}}-\delta_{1} & 0 & -2 x_{1} r_{1} a_{1}\left(T-T_{1}\right) e^{-a_{1}\left(T-T_{1}\right)^{2}} \\ 0 & r_{2} e^{-a_{2}\left(T-T_{2}\right)^{2}}-\delta_{2} & -2 x_{2} r_{2} a_{2}\left(T-T_{2}\right) e^{-a_{2}\left(T-T_{2}\right)^{2}} \\ \alpha_{1} & \alpha_{2} & -1\end{array}\right]$.

The corresponding eigenvalues for the extinction state $\left(\bar{x}_{1}=\bar{x}_{2}=\bar{T}=0\right)$ are:

$\lambda_{1}=r_{1} e^{-a_{1} T_{1}^{2}}-\delta_{1} ; \lambda_{2}=r_{2} e^{-a_{2} T_{2}^{2}}-\delta_{2} ; \lambda_{3}=-1$.

This extinction state is asymptotically stable if the three eigenvalues are simultaneously negative, which is satisfied if both $\left|T_{1}\right|>T_{1 c}$ and $\left|T_{2}\right|>T_{2 c}$.

The scability properties of the other equilibrium points can be similarly obtained. In particular, the two stationary states with $\bar{x}_{1}=0$ verify:

(i) $\left(\bar{x}_{1}=0, \bar{x}_{2}=\frac{T 2+T_{c 2}}{\alpha_{2}}, \bar{T}=T 2+T_{c 2}\right)$ is asymptotically scable if the next conditions simulcaneously occur:

$\left\{\begin{array}{l}\left|T_{2}+T_{c 2}-T_{1}\right| \geq T_{c 1} \\ T_{1}+T_{c 2} \geq 0 \\ \alpha_{2}>0\end{array}\right.$

(ii) $\left(\bar{x}_{1}=0, \bar{x}_{2}=\frac{T 2-T_{c 2}}{\alpha_{2}}, \bar{T}=T 2-T_{C 2}\right)$ is asymptotically stable if the next conditions simultaneously fulfill:

$$
\left\{\begin{array}{l}
\left|T_{2}-T_{c 2}-T_{1}\right| \geq T_{c 1} \\
T_{1}-T_{c 2} \geq 0 \\
\alpha_{2}<0
\end{array}\right.
$$

Similar stability conditions can be given for the two stationary states with $\bar{x}_{2}=0$.

It is worth remarking that there exist values of the optimal growth temperacures of the species for what the system can exhibit a triscability between extinction and selection of one of the two species. Otherwise, when either $T_{1}$ or $T_{2}$ is below their corresponding critical temperatures, $T_{c 1}$ and $T_{c 2}$, extinction $\left(\bar{x}_{1}=\bar{x}_{2}=\bar{T}=0\right)$ is unstable and then, the final selection of one of the two species depends on the initial conditions.

\subsection{The effect of extemal noise on the system dynamics}

As has been already mentioned, external noise could play a central role in system dynamics. Its influence on the global temperature $T$, as described in Eq. (3), allows the population to explore new dynamical ranges, making the system behavior richer than its deterministic counterpart. We will present in this section some simulations that clearly illustrate these effects. For a complete guide of how to solve numerically stochastic differential equations see (Milstein, 1995).

Noise is able to induce the survival of the unique species which, in the absence thereof, would die out. In Fig. 2, we show this situation for the one dimensional system (7) but now the differential equation for $T$ is given by:

$\dot{T}=\alpha x-T+\xi_{t}$

where $\xi_{r}$ is a Gaussian noise of intensity $\sigma$. The particular parameter values used are (notice that this parameter setup as well as the other chosen in this section, have no special meaning except that of allowing to depict the effect of noise on the deterministic behavior): (a)

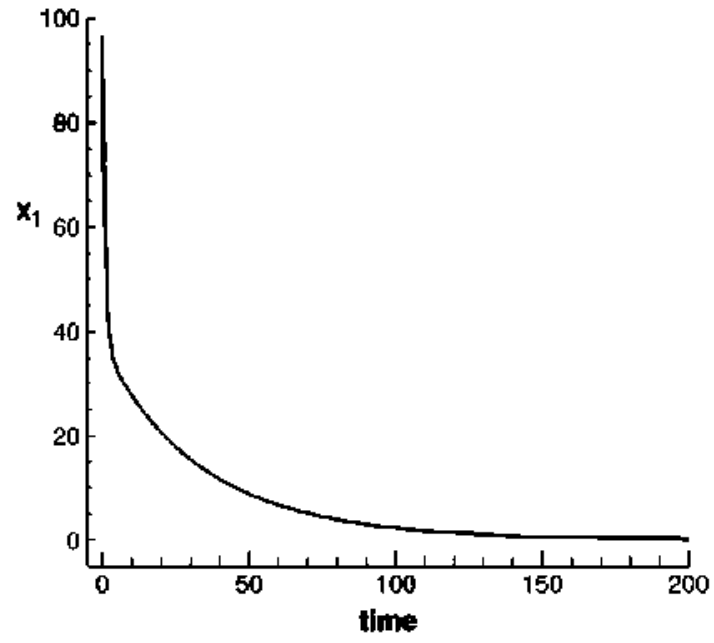

(b)

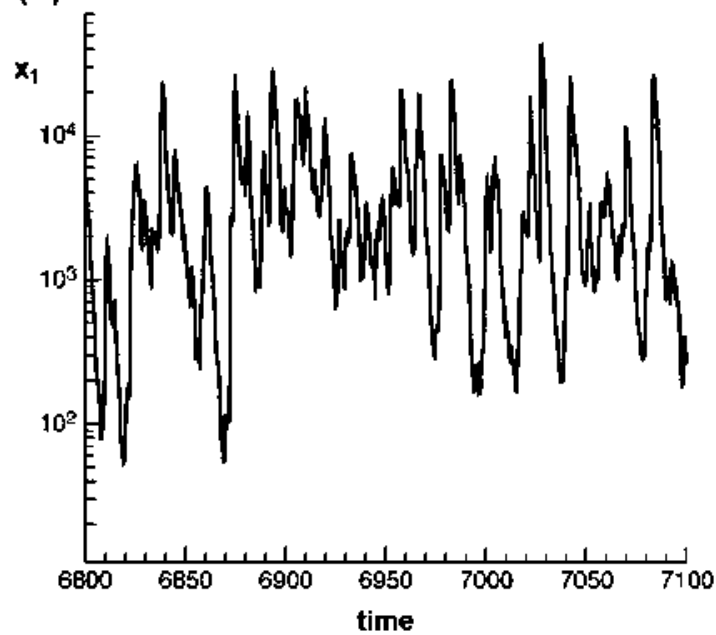

(c)

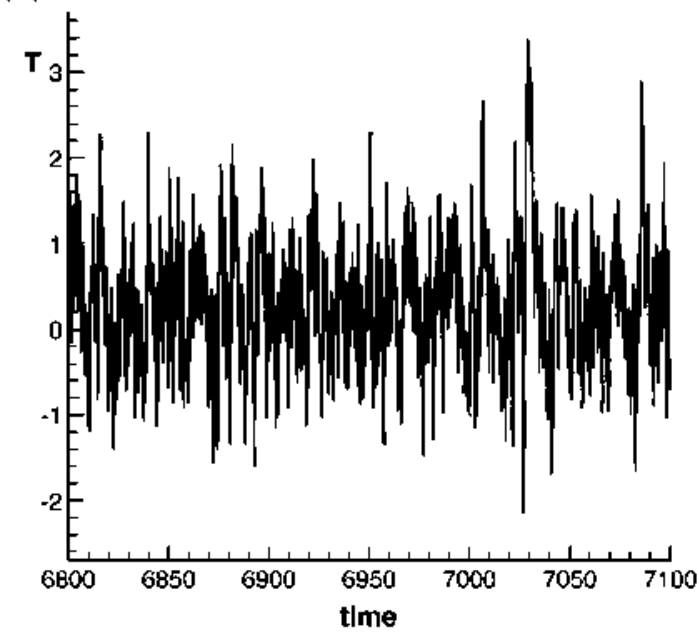

Fig. 2. Time evolution of the population $x$ of the unique replicator for: (a) null intensity of the noise and (b) and (c) a white noise of intensity $\sigma=1$. The time evolution of the temperature for the noisy system is depicted in (c). The rest of the parameter values ale stated in the main text As can be seen, the extinction of the species that occurs in (a) is avoided when an external white noise is perturbing the global temperature, $T$, (b). In order to appreciate better the dynamics only a short temporal window is depicted in (b) and (c). 
$r=5.2, a=0.5, T_{m}=-2, \delta=0.73, \alpha=10^{-4}$ and $\sigma=1$. The initial population is $x(0)=100$ and the initial temperature is $T(0)=1$. Meanwhile, for these parameter values, the deterministic stationary solution is $x=T=0$ (Fig. 2a). When a small intensity noise is affecting the global temperature the species population grows and remains fluctuating around a "safe" value (Fig. 2b). The time evolution of the global temperature is depicted in Fig. $2 \mathrm{c}$.

The same effect is obtained when two species are considered. Fig. 3 depicts two simulations for the following parameter values: $r_{1}=$ $r_{2}=5.2, a_{1}=a_{2}=0.75, T_{1}=-2, T_{2}=2, \delta_{1}=\delta_{2}=0.75, \alpha_{1}=10^{-3}$. $\alpha_{2}=-210^{-4}$ and $\sigma=2$. Initial conditions are $x_{1}(0)=x_{2}(0)=100$ and $T(0)=1$. Both populations vanish when noise is absent, but when an external white noise of intensity 2 is operating, then both species escape from extinction and coexist.

Noise can also be responsible for the coexistence of different species in situations in which deterministic competition always leads to selection of only one of the species. In Fig. 4 we show how the stochastic perturbation of the global temperature makes both species coexist far from extinction, although the deterministic dynamic leads to selection of $I_{1}$. Parameter values are: $r_{1}=r_{2}=5.2, a_{1}=0.55$, $a_{2}=0.75, T_{1}=-2, T_{2}=2, \delta_{1}=0.55, \delta_{2}=0.75, \alpha_{1}=10^{-3}, \alpha_{2}=$ $-210^{-4}$ and $\sigma=2$. The initial conditions are $x_{1}(0)=x_{2}(0)=100$ and $T(0)=1$.

These examples show the stabilizing power of this kind of uncorrelated noise. The effect of other types of correlated (colored) noise (Gardiner, 1995) in the dynamics of these adaptive systems is currently under study.

\section{An ecological model for simulating the evolution of public opinion}

As an application of this complex adaptive system we study the formation and evolution of public opinion, one of the most interesting problems ever posed in sociology (Schweitzer, 2003; Van Ginneken, 2003: Weidlich, 2000). Every society has a collective or public opinion that is formed from individual opinions according to complex and, hitherto, not well known rules. As an emergent phenomenon, public opinion changes over time as a result of variation of individual opinions as well as of external influences. The idea that cultural traits can be viewed as replicators (memes) was already stated by Dawkins (1976).

Here, opinions are assumed to be chains of $v$ digits that take different values, namely, YES (1) or NO (0), as obtained, for instance, from questionnaires. The solution space is then, formed by $2^{\nu}$ opinions. It is thought that each opinion (genome) contains two kinds of information: (i) its social influence $\left(\alpha_{i}\right)$ and (ii) its individual preferences $\left(T_{i}\right)$. Each opinion is able to replicate itself, giving rise to a population of opinions. The population size of each opinion $I_{i}$ is described by $x_{i}$. New opinions appear by mutations, e.g. by changing one or more digits of the template opinion.

It is assumed that public opinion can be measured by a single parameter, similar to a global temperature, $T$. It can be considered as an index that takes into account people preferences, weighted according to the influence of each individual. In principle, public opinion is accessible to the whole society, and thus influences the individual opinion. Therefore, both the global opinion $(T)$ and the individual preferences $\left(T_{i}\right)$ influence each other. Contrary to most of the models about evolution of opinion (Alves et al., 2002; Bagnoli et al., 2002; Tipan Verella and Wardak, 2008) our model does not explicitly consider local interactions (indeed, at present, contact with our neighbors is highly restricted). Instead, agents interaction is mediated by a public opinion that is broadcasted through mass media, mainly TV, newspapers and radio.

Each opinion has a fitness that depends on the difference between the individual, $T_{i}$, and the public opinion, T. Opinions close to the predominant opinion are more likely to spread through society. On the contrary, (a)

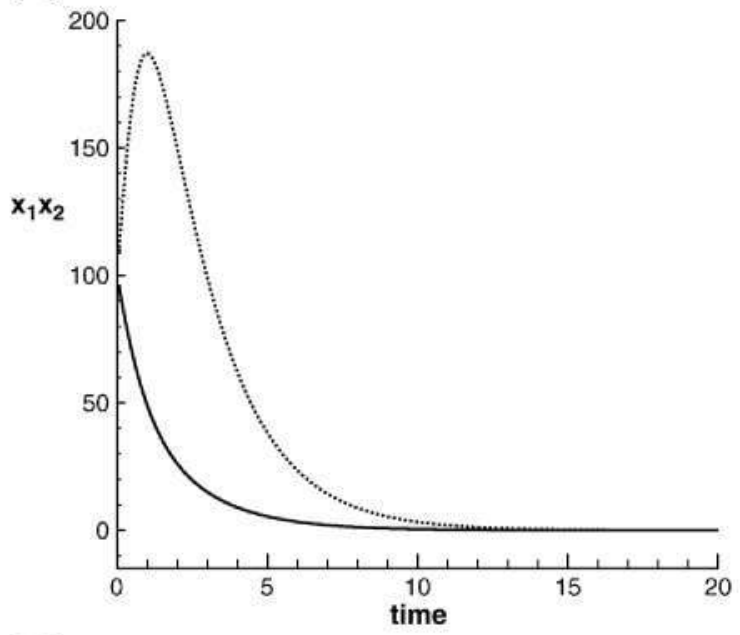

(b)

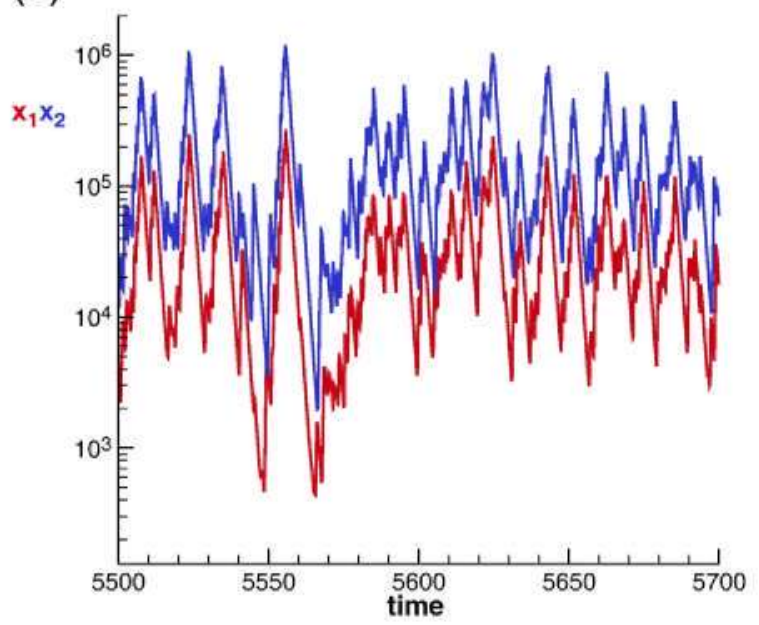

(c)

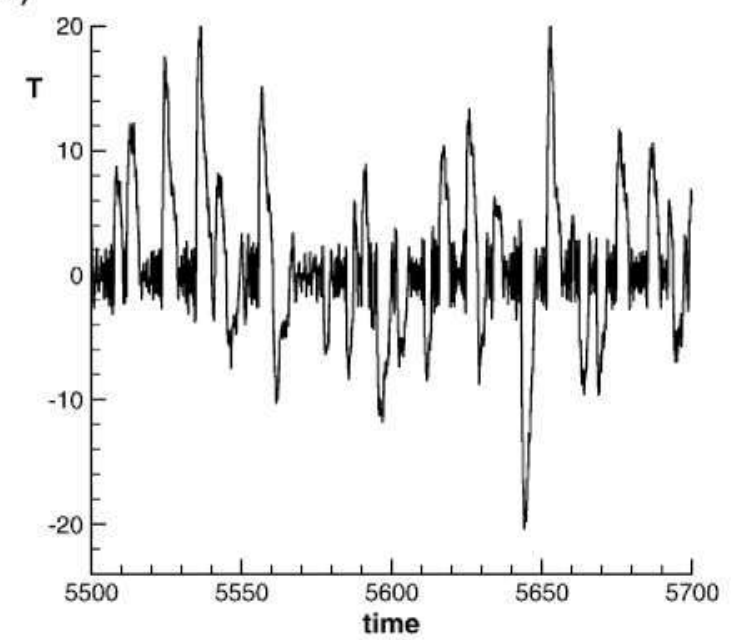

Fig. 3. Time evolution of the populations of the two species $x_{1}$ and $x_{2}$ for: (a) null intensity of the noise and (b) and (c) a white noise of intensity $\sigma=2$. The rest of the parameter values are stated in the main text. As can be seen in (b), the external noise is able to prevent the deterministic extinction of both species (a). The time evolution of the noisy temperature is also depicted in (c). As in the previous figure, a temporal window in the steady regime is depicted in (b) (species populations) and (c) (global temperature).

"distant" opinions have low survival probability. This behavior is well reflected by the fitness functions defined in Eq. (2). The total population is bounded, though no explicit population constraint is applied. 
(a)

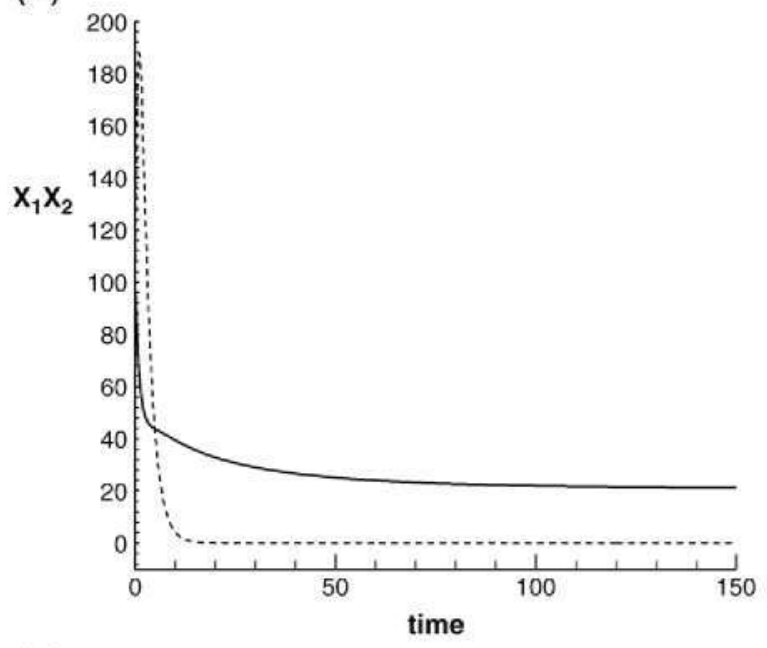

(b)

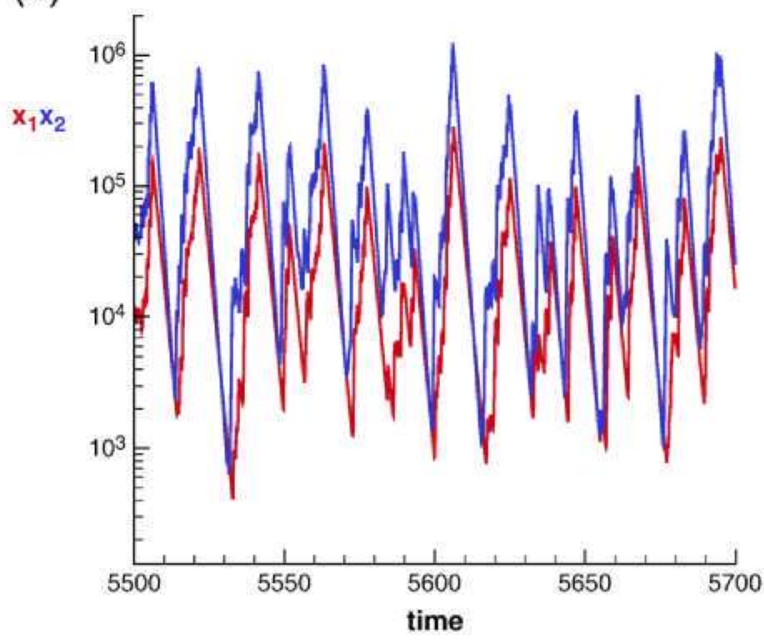

(c)

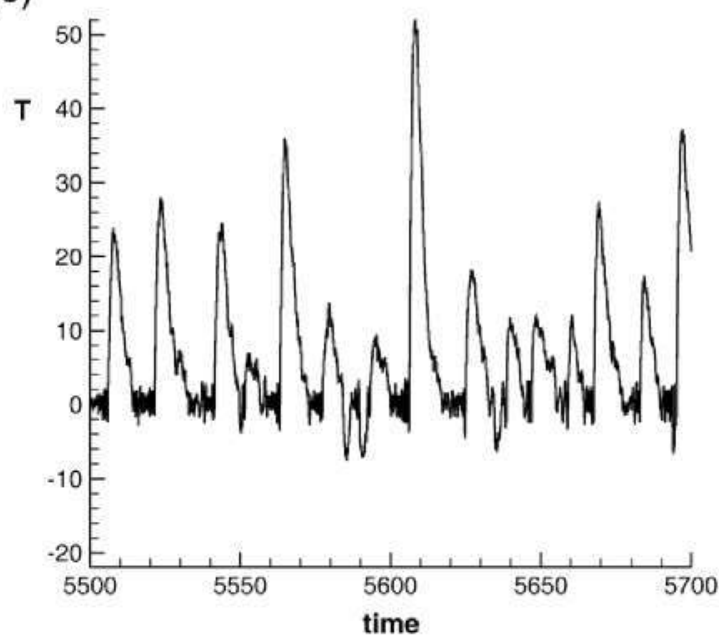

Fig. 4. Time evolution of the populations of the two species $x_{1}$ and $x_{2}$ for: (a) null intensity of the noise and (b) and (c) a white noise of intensity $\sigma=2$. The rest of the parameter values are shown in the main text. In (a) selection of one species occurs, whereas in (b) the presence of the external white noise allows the other species to escape from extinction and so, both species coexist. Notice the large variations of the global temperature in figure (c). As in the previous figures, to see better the system behavior we show a temporal window in the steady regime in (b) and (c).
The evolutionary algorithm is implemented in the following steps (see also the Fig. 5 for further details):

1. Generate at random an initial population $N(0)=\left\{I_{1}, \ldots, I_{N}\right\}$.

2. Evaluate the fitness of each individual $f_{i}=10 e^{-\left(T-T_{i}\right) 2}$ for $i=1,2, \ldots, N$.

3. Select a subset to reproduce, namely the fittest individuals.

4. Breed a new generation of solutions through digit mutation and give birth to offsprings.

5. Remove a percentage of the population according to the death rate.

6. Update the global temperature, $T$.

7. Repeat 2-6 steps until \{terminating condition\}.

A crucial point when simulating standard evolutionary algorithms is the mapping between the sequence space and the fitness landscape. The relevance of this assignment is even stronger in this adaptive quasispecies model since the fitness landscape changes over time as the population evolves. In order to show the potentialities of this model we have implemented the following assignment. The personal preferences $T_{i}$ of each opinion $I_{i}$ is the natural number codified by the first part of its binary sequence, normalized to the effective range $[-40,40]$. Similarly, opinion influence on $T, \alpha_{i}$, is the natural number codified by the second part of the binary sequence normalized to the range $[-0.1,0.1]$. It is worth remarking that, contrary to personal opinions $T_{i}$, public opinion as measured by $T$ is not restricted to vary in $[-40,40]$. Nonetheless, it is automatically self-regulated by the own population since a value of $T$ out of this range drastically reduces the fitness of every personal opinion (whose optimal growth rate is centered on $\left.T_{i}[-40,40]\right)$. We have considered the same fitness function for all opinions:

$f_{i}=10 e^{-\left(T-T_{i}\right)^{2}}$.

Notice that the variance of this Gaussian (a measure of its width) is 1. The evolution of this system on other fitness landscapes is currently under study and will be addressed elsewhere.

All simulations started with an initial population of 5000 randomly generated opinions. This gives rise to a random population of personal preferences $T_{i}$ and social influences $\alpha_{i}$. Initially, the public opinion $T$ is set to 0 . The public opinion at the next step is computed by a first order discretization of the differential Eq. (3) without any external noise, i.e.

$T(n+1)=(1-\Delta t) T(n)+\Delta t \sum_{k} \alpha_{k} N_{k}(n)$

The time step is $\Delta t=10^{-3}$. The death rate $\delta$ is selected so to assure a reasonable size of the total population. It is worth remembering that there is no additional constraint to the size of the total population. Nonetheless, it is internally self-regulated by the public opinion $T$ that limits the fitness of each individual (if $|T|>40$, then the fitness $f_{i}$ (Eq. (21)) drops drastically and prevents species self-replication). The total number of generations is left variable to adequately show the system behavior.

In the following subsection we study the behavior of this evolutionary model of public opinion by varying the mutation rate of both the personal preferences $T_{i}$ and the social influence on the public opinion $\alpha_{i}$. The study is not intended to be exhaustive but, on the contrary, to show a variety of relevant behavior patterns appearing under particular conditions. Each simulation is a realization of the system and can vary appreciably every run.

\subsection{The limit case of null mutation rate}

When the mutation rate is null, then selection only works among the species present in the initial population. In this case, the unique stationary state is extinction. Fig. 6(a) presents the time evolution of the public opinion $T$ (dashed line) and the average fitness (solid line), whereas the total population is depicted in Fig. 6(b). As can be seen, 


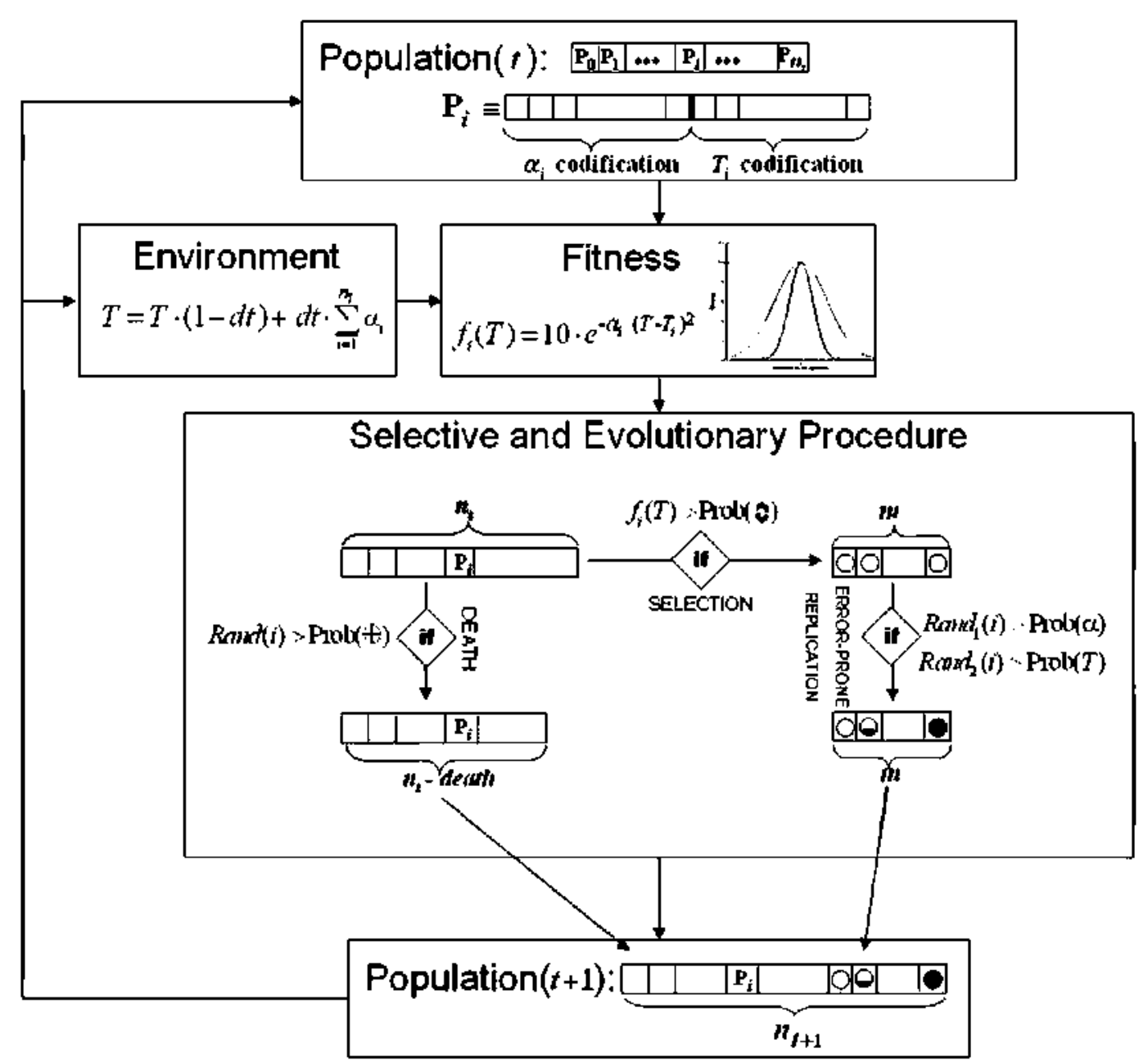

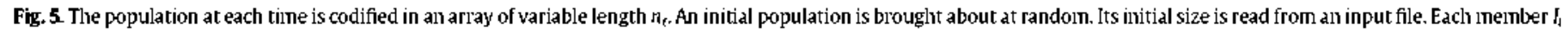

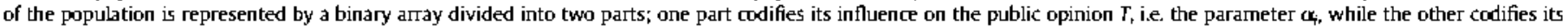

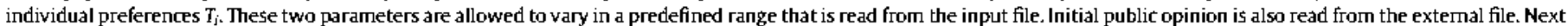

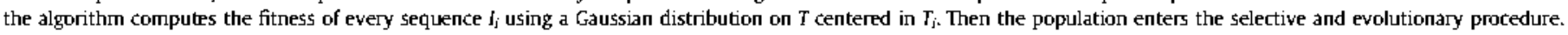

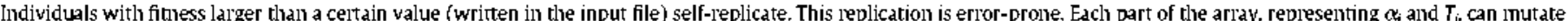

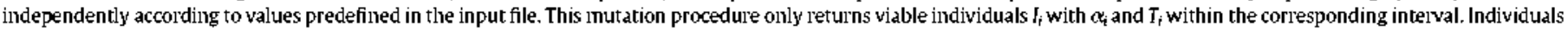

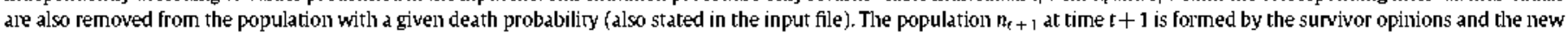
Imutant opinions. Public opinion $T$ is updated accolding to the first order discretization: $T_{5+1}=T_{5}(1-\Delta t)+\Delta t \sum_{k}{ }^{n_{i+1}+1} \alpha_{k}$.

after reaching a very high average fitness, the system starts to destabilize through a sharp increase of the population that suddenly ends in a drastic drop that causes its extinction. It turns out that the system has no capability to adjust itself to the variable environment that is changing as the population distribution varies. In the limit, when a fittest survival species remains, it drives the public opinion $T$ according to its own influence $(\alpha)$. But, as the population rises, public opinion is moving far from the particular opinion of the survival species, then the fitness of the fittest opinion tends to decrease and, finally, it provokes its own death. It is a kind of species suicide due to the lack of self-regulation (intrinsic extinction). This type of extinction is different from other kinds of extinctions, namely those caused by the depletion of natural resources or by external catastrophes.

\subsection{Exploring new opinions $T_{i}$ with an accurate $\alpha_{i}$-replication}

Now, let us explore the system behavior when only personal preferences can mutate. The species infuence is kept fixed (and randomly defined in the initial conditions). As can be seen in Fig. 7, the public opinion is brought to the limits for self-replication (in these simulations to $T=-40$ ). So, species with optimal growth temperature close to these artificial limits are selected. A quasispecies is formed around the public opinion $T=-40$ with all the individual opinions having the same $\alpha=-0.02636$. In this case, the system is not able to reach a high average fitness (around 8 over 10 ). This means that if $\alpha$ is error-free the effective optimization of personal preferences is prevented. Nevertheless, because no adjustment of the influence of species on global temperature is possible, the probability of extinction due to external perturbations is still relatively high.

\subsection{Exploting new infuences $\alpha_{i}$ with an eiror-fiee $T_{i}$-replication}

As a next step, we leave personal influences $\alpha_{i}$ to mutate whereas the species preferences $T_{i}$ are kept fixed. A population with a distribution of optimal growth preferences $T_{i}$ and influences $\alpha_{i}$ randomly generated at the beginning is left to evolve. A selective process occurs at the level of personal opinions $T_{i}$ that suddenly becomes fatal for the whole population. The population cannot respond to the induced changes in the environment and extinction becomes the final outcome (as occurred in Fig. 8).

\subsection{Quasispecies distribution of opinions}

The previous examples show that, in order to have an optimized set of opinions which, in turn, assures more stabilicy, i.e. a longer permanence time in the system, an efficient couple $\left\{T_{i}, \alpha_{i}\right\}$ is required. 
(a)

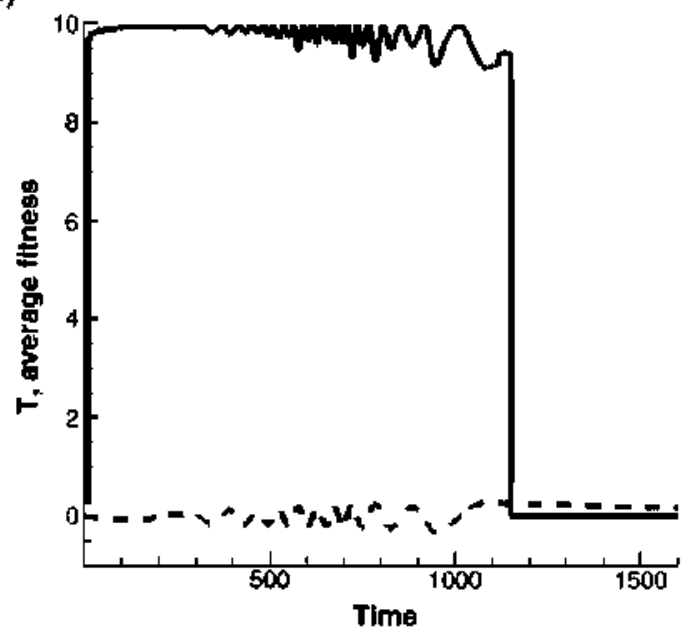

(b)

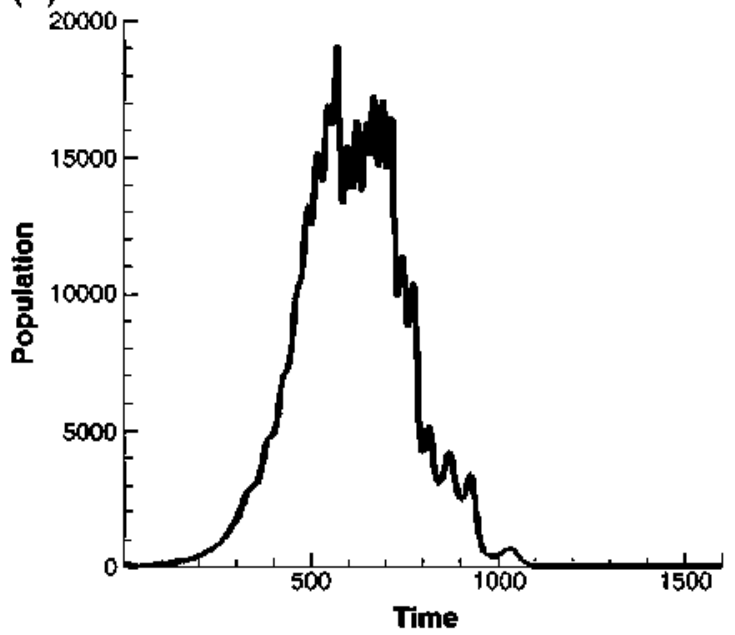

Fig. 6. In this simulation, opinions self-replicate accurately so new opinions, dillerent from that present in the initial population, cannot appear. So, the mutation ratrs for both $T$ and $\alpha$ are null, i.e. $M_{T}=0 \%$ and $M_{\alpha}=0 \%$. The time evolution of public opinion $T$ (dashed line) and the average fitness (solid line) is depicted in (a), whereas the total population is shown in (b). The death rate is $\delta=99 \%$

An optimal situation seems to be a low enough mucation rate for $T_{i}$ and a not too high mucation rate for $\alpha$. This choice allows an average fitness above 9 over 10 (for a large range of parameter values) to be obtained. However, the mutation rates must be carefully adapted for the opinion distribution to be stable. In this situation, the population is mainly formed by an opinion, surrounded by its closest mucants (the so called quasispecies distribution Eigen, 1971; Eigen et al., 1989). The interesting point here is that this quasispecies distribution is based on the personal preferences $T_{i}$ and has a substructure formed by the corresponding $\alpha$-values. This substructure allows the correct adaptation of the dominant opinion to the public opinion that has been escablished by this quasispecies. Fig. 9 depicts the quasispecies formed in a simulation for the mutation rates $M_{T}=5 \%$ and $M_{\alpha}=20 \%$. As can be seen, selection is operating on $T_{i}$-values (Fig. 9a). For these selected temperatures, a uniform distribution of $\alpha$ appears (Fig. 9b).

\section{Conclusion}

In this paper we have analyzed a dynamic model in which errorprone self-replicative species (replicators) interact with their environment. The model follows previous approaches by Lansing et al. (1998) and McDonald-Gibson et al. (2008) and can be understood as an extension of both the Daisyworld of Watson and Lovelock (a)

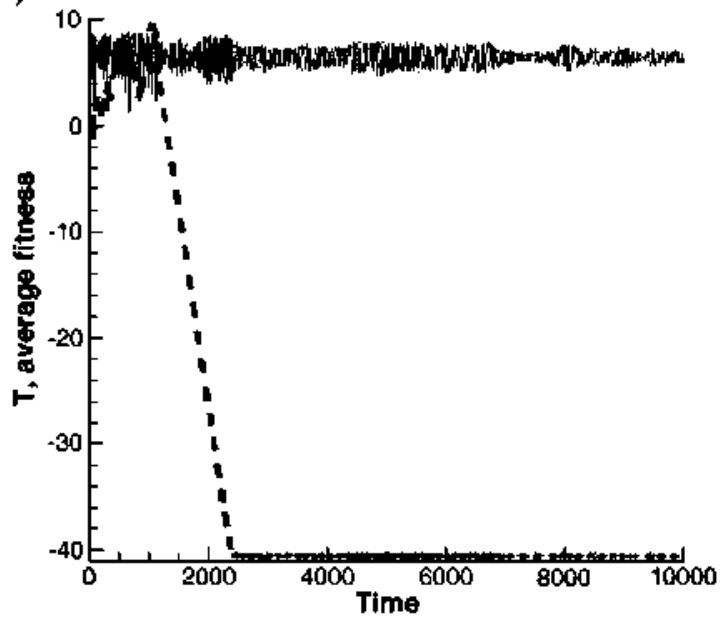

(b)

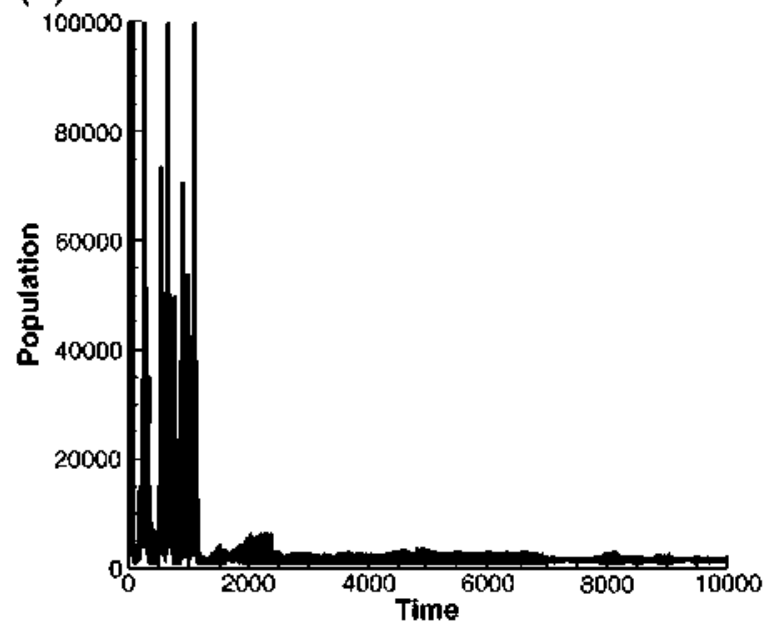

Fig. 7. A simulation obtained for a lull $\alpha$-1nutation rate, i.e. $M_{\alpha}=0$. The mutation rate of the personal preferences is equal for all species $M_{T}=10 \%$. As before (a) shows the time evolution of public opinion (dashed) and the average fimess (solid). whereas (b) depicts the time evolution of the total population. A quasispecies is formed around $T=$ $\mathbf{- 4 0}$, with all the species having a $\alpha$-value equal to -0.02636 , although the average fitness is not optimal (around 8 over 10.). Notice the large value of the total population that occurs during the transient period. The death rate is $\delta=65 \%$.

(Lovelock, 1992; Watson and Lovelock, 1983; Wood et al., 2008) and the quasispecies model of Eigen and Schuster (Eigen, 1971; Eigen et al., 1989). Even in the error-free limit, the mathematical model is too complex to be analytically solved. Nevertheless, a complete solution can be obcained in low-dimensional cases with null external noise. The effect of white noise on the system behavior has been also tackled in these low-dimensional cases by simulating the corresponding stochastic differential equations. The system dynamics becomes much richer when mutation is taken into account. An algorithm for simulating this system has been implemented and has been applied to study the formation and evolution of public opinion.

\section{Discussion}

Contrary to previous quasispecies models, where mutation operates on a single parameter, this model involves two system parameters that can mutate independently: the species optimal growth temperature $T_{i}$ and the species influence on the global temperature, $\alpha_{i}$. Note that both parameters have an effect on the species fitness. The existence of more than one error-prone species parameter yields a new type of quasispecies distribution as has been illustrate in Section 4.4. A structured quasispecies is formed when the 
(a)

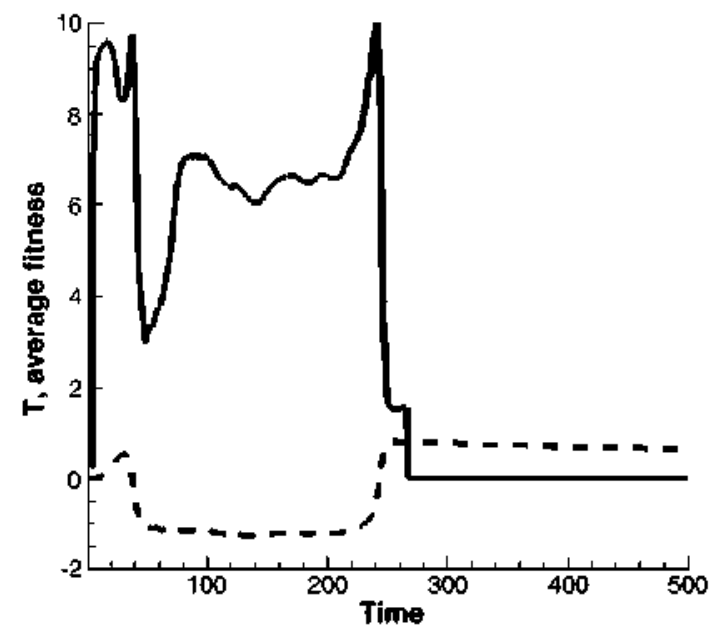

(b)

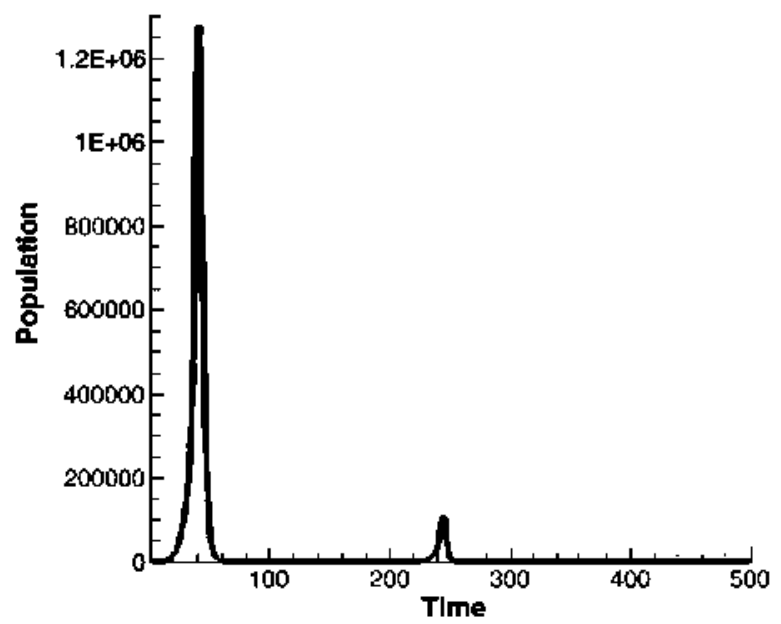

Fig. 8. Time evolution of (a) $T$ (dashed) and the average fimess (solid) and (b) the total population. Mutation rates are $M_{T}=0$ and $M_{c}=10 \%$. In this case, the system is not able to adapt itself to the changing environment and therefore, the only final outcome is total extinction. In the beginning, a selective process occurs and relatively good opinions remain. Under these good conditions, these opinions self-replicate very quiclely and the whole population increases to very large values. This causes a drastic decrease in public opinion which causes a sharp drop in total population size until it vanishes completely. The death rate is $\delta=65 \%$.

mutation rate for the optimal growth temperature is low enough and the mucation rate on $\alpha$ is non-null: for each optimal temperature $T_{\text {; }}$ a distribution of $\alpha_{j}$ appears, thus allowing the system to adapt better to a changing environment. This aspect has been investigated in more detail for its possible applications on evolutionary optimization algorithms (Forrest, 1993; Goldberg, 1989; Perales-Gravan and Lahoz-Beltra, 2008).

However, the quasispecies obcained in this kind of dynamic landscapes are not longer stable. Since there is always a non-null probability of appearance of a new mutant, and this new mutant can have a significant influence on the global temperature, i.e. modify the fitness landscape, then the current wild rype and its nearest mucants can be displaced by others best adapted to the new fitness landscape. The fitness of each species does not possess any information about the chances of survival in the future (Brookfield, 2001). In this new scenario, the permanence time of a sequence becomes a fundamental characteristic that deserves further attention. This issue is related to the theory of survival analysis, one of the most fruitful fields in mathematical statistics (see, for instance, Ma and Krings, 2008).

It turns out that this kind of models mimics reasonably well the formation and evolution of collective opinions. This is an old issue that (a)

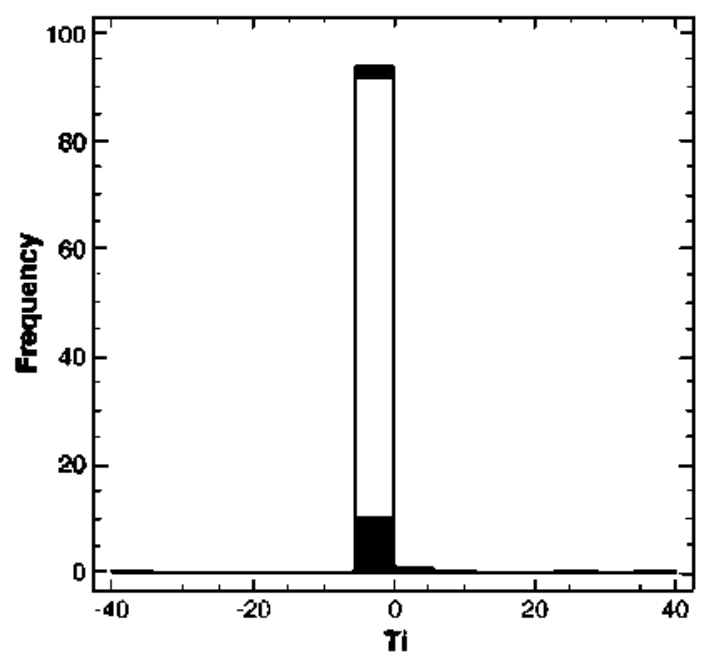

(b)

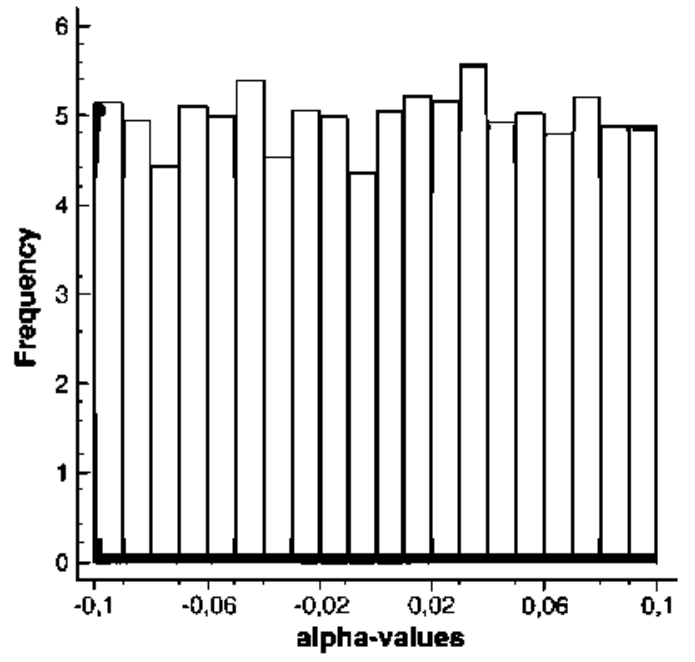

Fig 9. When the inutation rate of both the personal preferences, $T_{i}$, and its influence on the public opinion, $\alpha$, are non-null, then the system is capable of adapting to the changing public opinion that the whole set of individual opinions has brought about In this simulation the mutation rates are: $M_{\mathrm{T}}=5 \%$ and $M_{\alpha}=20 \%$ and the death rate is $\delta=65 \%$. The distribution of species preferences $T_{j}$ is shown in (a), whereas the distribution of $\alpha$-values is depicted in (b). As can be seen, selection is operating on $T_{i^{-}}$ values. For these selected temperatures, a uniform distribution of $\alpha$ appears.

was already treated as an evolutionary problem by Richard Dawkins in his famous "The Selfish Gene" (Eigen, 1971) where the term "meme" was introduced. Since then, this problem has been addressed from different viewpoints: psychological, sociological, economical and, more recently, sociophysical (Castellano et al., 2009). Public opinion is another example of complex adaptive systems (CAS), where an emergent behavior (public opinion) appears as a consequence of individual or local interactions (Miller and Page, 2007; Van Ginneken, 2003). In models of collective opinions based on cellular automata individuals interact with their neighbors (Alves et al., 2002; Bagnoli et al., 2002; Tipan Verella and Wardak, 2008). Under this approach, individuals are modelled as cells, the interaction between people is governed by micro-rules, and the spread of opinions is seen as a diffusive process. In contrast, we have shown that emergent phenomena can occur also when individuals interact indirectly through a shared quantity such as the collective or public opinion. Actually, individuals affect the environment via their opinions, and doing so they change the context in which their own opinion is 
weighted. As a result, the fitness of each opinion is a temporal variable that changes with population structure.

Assuming that noise is Gaussian and white (uncorrelated) implies that fluctuations in the temperature are the fastest process in the system. Memory effects in the global temperature $T$ could be modelled by a Gaussian noise with a non-zero correlation time (i.e. a OrnsteinUhlenbeck process (Gardiner, 1995)). This would allow a more realistic coupling between the global temperature and the population. Another possible generalization, inspired by the application to social sciences, is the study of the effect that an isolated sudden change in the conditions can have on the system dynamics. It is reasonable to think of situations in which an isolated evenc, such as a social scandal or a terrorist atcack. can drastically alter public opinion. A possible way of modelling these situations is by adding a shot noise in the differential equation of the global temperature causing sudden jumps at random times following a Poisson distribution. Work in this direction is in progress.

\section{Acknowledgments}

This work has been partially supported by project FIS2009-13690 of the Ministerio de Ciencia e Innovation de España and grant Q0G0120012 of the Universidad Politêcnica de Madrid. Rafael LahozBeltra was supported by the Laboratorio de Bioinformatica, Universidad Complutense de Madrid (UCM), and in part under the grant "Profesores UCM en el Extranjero 2008". We wish to thank Cormac de Brun for reading the manuscript and perfecting the English language. Finally, the authors gratefully acknowledge the anonymous reviewers for their suggestions which helped to improve this paper.

\section{References}

Avves, S.G., Oliveira Neto, N.M., Martins, M.L, 2002. Electoral surveys influence on the voting processes: a cellular automata model. Physica A 316, 601-614.

Bagnoli, F, Franci, F., Reclitman, R., 2002. Opinion formation and phase transitions in a probabilistic cellular automaton with two absorbing states. ACRI. : In: Bandini, S., Chopard, B., Tolnassini, M. (Eds.), LNCS 2493. Springer-Verlag, pp. 249-258.

Bak, P., Sneppen, K., 1993. Punctuated equilibrium and cliticality in a simple model of evolution. Phys. Rev. Lett 71, 4083-4086.

Brookfield, J.F.Y, 2001. Predicting the future. Nature 411, 999.

Castellano, C., Fortunato, S., Loreto, V., 2009. Statistical physics of social dynamics. Rev. Mod. Plys. 591-646.

Dawkins R. The selfish gene. Oxford University Press. 30th amiversaly edition 2006 (first published in 1976).

Eigen, M., 1971. Self-organization of matter and the evolution of biological macromolecules. Die Naturwiss. 58, 465-523.
Eigen, M., Schuster, P., 1979. The Hypercycle: A Plinciple of Natural Self-organization. Springer-Verlag.

Eigen, M., McCaskill, J., Schuster, P., 1989. The molecular quasi-species. Adv, Chem. Phys. 75, 149-263.

Forrest, S., 1993. Genetic algorithms: principles of natural selection applied to computation. Science 261 (5123). 872-878 (Aug 13).

Gardiner, C.W., 1995. Handbook of Stochastic Methods for Physics, Chemistry and the Natural Sciences, Springer Verldg.

Goldberg. D.E., 1989. Genetic Algorithms in Search, Optimization and Machine Learning. Addison-Wesley.

Hirsch, M.W., Sinale, S., 1974. Differential Equations, Dynamical Systems and Linear Algebra. Academic Press.

Hogeweg, P. 2007. From population dymanics to ecoinformatics: ecosystems as inultilevel information processing systems. Ecolog. Inform. 2, 103-111.

Lansing, J.S. Kremer, J.N., Smuts, B.B., 1998. System-dependent selection, ecological feedback and the energence of functional stiucture in ecosystems. J. Theor, Biol. $192,377-391$

Lovelock, J.E., 1992. A numerical model for biodiversiry. Philos. Trans. R. Soc. Lond. B Biol. Sci. 338, 365-373.

Ma, Zhanshan (Sam), Krings, Axel W., 2008. Survival Analysis Approach to Reliability. Survivability and Prognostics and Health Mandgenent (PHM). IEEE.

McDonald-Gibson, J., Dyke, J.G., Di Paolo, E.A, Harvey, I.R., 2008. Environmental regulation can arise under minimal assumptions. J. Theor. Biol. 251, 653-666.

Miller, J.H., Page, Scott E., 2007. Complex Adaptive Systems: An Introduction to Computational Models of Social Life. Princeton Univ Press.

Milstein, G.N., 1995. Numerical Integration of Stochastic Differential Equations. Kluwer Academic Press.

Nilsson, M., Snoad, N., 2000. Error thresholds for quasispecies on dynamic fitness landscapes. Phys. Rev, Letr. 84.

Perales-Gravan, C., Lahoz-Beltra, R, 2008. An AM radio receiver designed with a genetic algorithm based on a bacterial conjugation operator. IEEE Tralns. Evol. Comput. 12 (2), 129-142.

Rauch, E.M. Sayama, H., Bar-Yan, Y., 2002. Relationship between measures of fitness and time scale in evolution. Plys. Rev. Lett. 228101.

Saakian, D.B., Hu, C.-K., 2006. Exact solution of the Eigen model with general fitness functions and degradation rates. PNAS 103, 4935-4939.

Saunders, P.T., 1994. Evolution without natural selection: further implications of the Daisyworld parable. J. Theor. Biol. 166, 365-373.

Scluster, P., Sigmund, K., 1983. Replicator dynamics. J. Theor, Biol, 100, 533-538.

Schweitzer, F., 2003. Brownian Azents and Active Particles: Collective Dynamics in the Natural and Social Sciences. Spininger.

Tipan Verella, J., Wardak, A., 2008. Modeling public opinion and voting as a complex system with agent-based simulations. Proceedings of the 2008 IEEE Systems and Infolnnation Engineering Design Sylnposium, University of Virginia, Charlottesville, VA, USA, Pp. 261-266.

Van Gimeken, J., 2003. Collective Behavior and Public Opinion. Rapid Shifts in Opinion and Colminunication. Lawrence Erlbaum Associates, Inc.

Watson, A.J., Lowelock, J.E., 1983. Biological homeostasis of the global environment: the parable of Daisyworld. Tellus B 284-289.

Weidlich, W. 2000. Sociodynamics: A Systematic Approach to Mathematical Modelling in the Social Sciences. Harwood Academic Publishers.

Whighain, PA., Fogel, G.B., 2006. In: Recknagel, F. (Ed.), Ecological Applications of Evolutionary Computation in Ecological Informatics. Springer Verlag.

Wood, A.J., Ackland, G.J., Dyke, J.G., Williams, H.T.P., Lenton, T.M., 2008. Daisyworld: a review. Rev. Geophys. 46, RG1001. 